\title{
GAMMA-GLUTAMYL TRANSFERASE AS AN INDICATOR OF OBESITY: A CROSS-SECTIONAL STUDY
}

\author{
VINODHINI VM*, SUDHAN KB \\ Department of Biochemistry, SRM Medical College Hospital and Research Centre, SRM University, Kattankulathur, Tamil Nadu, India. \\ Email: vinodhini239@gmail.com
}

Received: 06 July 2016, Revised and Accepted: 07 September 2016

\section{ABSTRACT}

Objective: Obesity, characterized by an increase in excessive fat accumulation, represents a social problem worldwide and has been recognized as a major underlying factor in the pathogenesis of several diseases. Gamma-glutamyl transferase (GGT) is a cell-surface protein contributing to the extracellular catabolism of glutathione. Elevated GGT is strongly associated with obesity and excess deposition of fat in the liver, termed nonalcoholic fatty liver disease, which is thought to cause hepatic insulin resistance and contribute to the development of systemic insulin resistance and hyperinsulinemia. Therefore, we have investigated the serum GGT levels in obese individuals and the correlation of serum GGT with body mass index (BMI) and waist circumference.

Methods: The study was carried out in 100 obese patients and 100 non-obese individuals.

Results: Patients with obesity showed a significant increase in GGT levels when compared to the control group. The mean levels of BMI, WC, total cholesterol, triglycerides, low-density lipoprotein-cholesterol (LDL-c), very LDL-cholesterol, total protein and aspartate aminotransferase were found to be significantly elevated in the obese individuals compared to controls. The mean levels of high-density lipoprotein-cholesterol showed a significant decrease in the obese participants.

Conclusion: Elevated liver enzymes, although in normal ranges, especially at upper quartiles as observed in our study, may play a central role in early diagnosis of fat overflow to the liver. The findings of our study suggest that serum GGT levels may be a simple and reliable marker of visceral fat accumulation.

Keywords: Diabetes mellitus, Gamma-glutamyl transferase, Insulin resistance, Metabolic syndrome, Obesity.

(C) 2016 The Authors. Published by Innovare Academic Sciences Pvt Ltd. This is an open access article under the CC BY license (http://creativecommons. org/licenses/by/4. 0/) DOI: http://dx.doi.org/10.22159/ajpcr.2016.v9s3.13939

\section{INTRODUCTION}

Obesity, characterized by an increase in excessive fat accumulation, represents a social problem worldwide and has been recognized as a major underlying factor in the pathogenesis of several diseases [1] In a developing country like India, obesity and malnutrition are two ends of a spectrum: Obesity being an emerging issue which needs close monitoring [2].

Obesity also involves a growing number of children in developed countries. Studies have assessed that children who became obese in an early age were more likely to be obese as adults [3].

Recently, it has also been found that obesity is associated with lowgrade chronic systemic inflammation in adipose tissue. This condition is influenced by the activation of the innate immune system in adipose tissue that promotes the pro-inflammatory status and oxidative stress (OS), triggering a systemic acute-phase response. Several chronic diseases such as metabolic syndrome, diabetes mellitus, liver and cardiovascular diseases and cancer are also the result of obesity and associated with OS. It has been hypothesized that inflammation of adipose tissue in obese patient plays a critical role in the pathogenesis of obesity-related complications [4].

Gamma-glutamyl transferase (GGT) is a cell-surface protein contributing to the extracellular catabolism of glutathione (GSH). The enzyme is produced in many tissues, but most GGT in serum is derived from the liver [5]. Measurement of GGT in serum appears to be a sensitive index with low specificity in the diagnosis of alcoholics with hepatitis when correlated clinically [6]. GGT has a pivotal role in the maintenance of intracellular antioxidant defenses through its mediation of extracellular GSH transport into most types of cells [7].
Elevated GGT is strongly associated with obesity and excess deposition of fat in the liver, termed non-alcoholic fatty liver disease, which is thought to cause hepatic insulin resistance and contribute to the development of systemic insulin resistance and hyperinsulinemia [8].

Several mechanisms for GGT leakage are possible and include an increase in oxidative stress, proteolysis, glycosylation, GGT synthesis and endothelial damage [9]. The interrelations between serum GGT, obesity, other metabolic disturbances and plasma insulin raise the possibility that elevated GGT levels can help predict the development of metabolic syndrome and Type 2 diabetes [10]. Therefore, we have investigated the serum GGT levels in obese individuals and the correlation of serum GGT with body mass index (BMI) and waist circumference (WC) in obesity.

\section{METHODS}

The study protocol was performed in accordance with the approval of the Institutional Ethics Committee (ECN: 731/IEC/2015) and informed written consent was taken from all subjects.

\section{Patient selection}

Inclusion criteria

A total of 100 obese patients (BMI $\geq 25 \mathrm{~kg} / \mathrm{m}^{2}$ ); all the patients were in the age group of 18-45 years and the study included both males and females.

\section{Exclusion criteria}

Patients with diabetes mellitus, pregnancy, high alcohol consumption, known liver or kidney diseases and patients on medications which are enzyme inducers. 100 apparently healthy non-obese individuals (BMI 
$18-22.9 \mathrm{~kg} / \mathrm{m}^{2}$ ), without diabetes, hypertension, renal disease and any other systemic illness were selected for the study and they formed the control group.

\section{Sample collection}

Venous blood was collected from all the participants after an overnight $12 \mathrm{hrs}$ fast. $2 \mathrm{ml}$ of the blood sample was collected in an oxalate fluoride Vacutainer for estimation of fasting plasma glucose. $3 \mathrm{ml}$ of blood collected in a plain Vacutainer was allowed to clot and serum was separated by centrifugation at 3000 RPM for 10 minutes.

Plasma glucose (FBS), total cholesterol, triglycerides, high-density lipoprotein-cholesterol (HDL-c), low-density lipoprotein-cholesterol (LDL-c), total protein, aspartate aminotransferase (AST), alanine transaminase (ALT) and GGT were measured by using standard kits in Beckmann Coulter auto analyzer on the same day of sample collection.

\section{RESULTS}

Comparison was made between the two groups using student's $t$-test.

Patients with obesity showed a significant increase in GGT levels $(25.42 \pm 9.5$ vs. $17.52 \pm 7.05 ; \mathrm{p}<0.001)$ when compared to the control group.

The mean levels of BMI, WC, total cholesterol, triglycerides, LDL-c, Very LDL (VLDL-c), total protein and AST were found to be significantly elevated in the obese individuals compared to controls. The mean levels of HDL-c showed a significant decrease in the obese participants (Table 1).

GGT levels in obese individuals were found to correlate positively with serum triglycerides $(\mathrm{r}=0.4236, \mathrm{p}<0.001)$ (Table 2$)$.

GGT levels in obese individuals were found to correlate positively with BMI ( $r=0.1036, p=0.4288$ ) (Table 3).

GGT levels in obese individuals patients were found to correlate positively with WC $(\mathrm{r}=0.0445, \mathrm{p}=0.7670)$ (Table 4).

\section{DISCUSSION}

GGT is central in GSH hemostasis which is an important antioxidant defense for the cell. Therefore, GGT plays an important role in antioxidant defense systems. Elevated GGT levels could be a marker of oxidative stress and subclinical inflammation.

Elevated GGT is strongly associated with obesity and excess deposition of fat in the liver, termed non-alcoholic fatty liver disease, which is thought to cause hepatic insulin resistance and to contribute to the development of systemic insulin resistance and hyperinsulinemia [11]. Increased serum concentration of GGT could identify people with low but persistent increase of oxidative and other cellular stresses [12].

In our study, obese individuals have shown a significant increase in serum GGT levels when compared to the non-obese control group. At the same time, we have observed that the serum GGT levels in the obese group are in the high normal range only.

Recent population-based epidemiological studies have shown a strong association of serum GGT activities within the reference interval with many cardiovascular disease risk factors or components of metabolic syndrome. In addition, in prospective studies, baseline serum GGT activity predicted future diabetes, hypertension, stroke and myocardial infarction. Among these diseases, serum GGT within the reference interval most strongly predicted incident Type 2 diabetes [13].

Three prospective cohort studies found an interaction, not statistically significant, in which obesity was weakly associated with incident diabetes in people with low-normal serum GGT but strongly associated in those with high normal serum GGT [14].
Table 1: Comparison of mean $\pm S D$ of the measured biochemical parameters between the control and obese groups and the statistical significance of the differences

\begin{tabular}{llll}
\hline Parameters & $\begin{array}{l}\text { Control } \\
\text { (n=100) }\end{array}$ & $\begin{array}{l}\text { Obese } \\
\text { individuals } \\
\text { (n=100) }\end{array}$ & p \\
\hline BMI (Kg/m ${ }^{2}$ ) & $19.84 \pm 1.26$ & $29.07 \pm 1.98$ & $<0.001$ \\
WC (cm) & $77.36 \pm 4.25$ & $102.48 \pm 6.38$ & $<0.001$ \\
GGT (U/L) & $17.52 \pm 7.05$ & $25.42 \pm 9.5$ & $<0.001$ \\
T. protein (g/dl) & $7.10 \pm 0.53$ & $7.30 \pm 0.50$ & 0.0063 \\
AST (U/L) & $23.64 \pm 7.80$ & $27.22 \pm 10.40$ & 0.0064 \\
ALT (U/L) & $24.71 \pm 15.50$ & $25.28 \pm 14.80$ & 0.7906 \\
Total cholesterol (mg/dl) & $140.33 \pm 17.77$ & $194.27 \pm 24.83$ & $<0.001$ \\
Triglycerides (mg/dl) & $108.35 \pm 45.94$ & $155.23 \pm 80.06$ & $<0.001$ \\
HDL-c (mg/dl) & $42.99 \pm 7.47$ & $32.66 \pm 7.24$ & $<0.001$ \\
LDL-c (mg/dl) & $92.28 \pm 22.42$ & $134.79 \pm 27.49$ & $<0.001$ \\
VLDL-c (mg/dl) & $22.66 \pm 9.62$ & $32.42 \pm 16.43$ & $<0.001$ \\
FPG (mg/dl) & $93.81 \pm 7.66$ & $94.19 \pm 5.44$ & 0.6863 \\
\hline
\end{tabular}

Values are expressed in mean \pm standard deviation. The values are statistically significant if the $\mathrm{p}<0.05$, GGT: Gamma-glutamyl transferase, SD: Standard deviation, BMI: Body mass index, WC: Waist circumference, AST: Aspartate aminotransferase, ALT: Alanine transaminase, HDL-c: High-density

lipoprotein-cholesterol, LDL-c: Low-density lipoprotein-cholesterol, VDL-c: very density level-cholesterol

Table 2: The Pearson's correlation analysis between GGT levels and triglycerides in obese individuals

\begin{tabular}{lll}
\hline Parameters & Mean \pm SD & r (p) \\
\hline GGT (U/L) & $25.42 \pm 9.5$ & $\mathrm{r}=0.423(<0.001)$ \\
Triglycerides $(\mathrm{mg} / \mathrm{dl})$ & $155.23 \pm 80.06$ & \\
\hline
\end{tabular}

GGT: Gamma-glutamyl transferase, SD: Standard deviation

Table 3: The Pearson's correlation analysis between GGT levels and BMI in obese individuals

\begin{tabular}{lll}
\hline Parameters & Mean \pm SD & r (p) \\
\hline GGT $(\mathrm{U} / \mathrm{L})$ & $25.42 \pm 9.5$ & $\mathrm{r}=0.1036(0.4288)$ \\
$\mathrm{BMI}\left(\mathrm{Kg} / \mathrm{m}^{2}\right)$ & $29.07 \pm 1.98$ & \\
\hline
\end{tabular}

GGT: Gamma-glutamyl transferase, BMI: Body mass index, SD: Standard deviation

Table 4: The Pearson's correlation analysis between GGT levels and WC in obese individuals

\begin{tabular}{lll}
\hline Parameters & Mean \pm SD & r (p) \\
\hline GGT $(\mathrm{U} / \mathrm{L})$ & $25.42 \pm 9.5$ & $\mathrm{r}=0.0445(0.7670)$ \\
WC $(\mathrm{cm})$ & $102.48 \pm 6.38$ & \\
\hline
\end{tabular}

GGT: Gamma-glutamyl transferase, WC: Waist circumference, SD: Standard deviation

Moreover, Wannamethee et al. revealed that elevated levels of ALT and GGT within the normal ranges are found to be the independent predictors of Type 2 diabetes mellitus. Hence, elevated liver enzymes, although in normal ranges, especially at upper quartiles as observed in our study, may play a central role in early diagnosis of fat overflow to the liver [15].

BMI, WC and serum triglycerides are components of metabolic syndrome. We observed that the GGT levels in obese individuals of our study were found to correlate positively with BMI. Our results are consistent with the findings of Puukka et al., who observed a positive correlation between GGT and BMI measured in 2490 participants [16].

WC is used as a measure of abdominal adiposity because a correlation between WC and the amount of intra-abdominal fat has been observed 
with computed tomography [17]. In our study, the GGT levels in obese individuals correlated positively with WC. The GGT levels in obese individuals were also found to correlate positively with serum triglycerides and this association was found to be statistically significant.

Rantala et al. and Sakugawa et al. investigated the relationship between GGT and metabolic syndrome and found that the serum GGT levels correlated with components of metabolic syndrome. Hence, estimation of GGT levels can be considered in the algorithm for metabolic syndrome [18]. Pangaluri et al. found metabolic syndrome to be associated with subclinical hypothyroidism and compounded cardiovascular risk factors [19].

A prospective cohort study involving 7458 non-diabetic men has concluded that a raised serum GGT is an independent risk factor for diabetes mellitus. In a study including 6217 Japanese men, Nakanishi et al. indicated that serum GGT may be an important predictor for developing metabolic syndrome and Type 2 diabetes [10].

A number of recent studies have suggested that abnormal hepatocellular function is associated with obesity, insulin resistance and Type 2 diabetes [20]. Elevation of serum GGT could be an expression of excess fat deposited in the liver, which is regarded as a feature of the insulin resistance syndrome.

We found the mean levels of total cholesterol, triglycerides, LDL-c and very LDL-cholesterol (VLDL-c) to be significantly elevated in the obese individuals compared to controls. The mean levels of HDL-c showed a significant decrease in the obese participants.

Various studies suggested that elevated serum GGT could be the expression of subclinical inflammation which also contributes to the development of Type 2 DM. Gohel and Chacko have found a statistically significant positive linear relationship between serum GGT and hsCRP in patients with Type 2 DM [12]. Elevated liver enzymes may reflect inflammation, which impairs insulin signaling both in the liver and systemically [21]. Vinodhini et al. have reported the presence of elevated hsCRP concentrations in obese females with polycystic ovary syndrome [22]. As serum GGT is highly associated with WBC count and some features of low-graded inflammation, an elevated GGT could be the expression of subclinical inflammation or an insulin-resistant state [23]

\section{CONCLUSION}

We have observed a comparative increase in serum GGT levels in obesity which was found to correlate positively with serum triglycerides, BMI and WC. The findings of our study suggest that serum GGT levels may be a simple and reliable marker of visceral fat accumulation.

\section{REFERENCES}

1. Sikaris KA. The clinical biochemistry of obesity. Clin Biochem Rev 2004;25(3):165-81.

2. Remesh A. Obesity: Pathophysiology and management-a pharmacological perspective. Asian J Pharm Clin Res 2013;6(1):11-3.

3. Freedman DS, Wang J, Thornton JC, Mei Z, Sopher AB, Pierson RN Jr, et al. Classification of body fatness by body mass index-for-age categories among children. Arch Pediatr Adolesc Med 2009;163(9):805-11.

4. Xu H, Barnes GT, Yang Q, Tan G, Yang D, Chou CJ, et al. Chronic inflammation in fat plays a crucial role in the development of obesityrelated insulin resistance. J Clin Invest 2003;112(12):1821-30.
5. Emdin M, Pompella A, Paolicchi A. Gamma-glutamyltransferase, atherosclerosis, and cardiovascular disease: Triggering oxidative stress within the plaque. Circulation 2005;112(14):2078-80.

6. Vasantha M, Gayathri B. Level of serum gamma glutamyl transferase in alcoholic hepatitis as a diagnostic marker. Int J Pharm Bio Sci 2016;7(1):(B)9-11.

7. Karp DR, Shimooku K, Lipsky PE. Expression of gamma-glutamyl transpeptidase protects ramos B cells from oxidation-induced cell death. J Biol Chem 2001;276(6):3798-804.

8. Lazarin Mde O, Ishii-Iwamoto EL, Yamamoto NS, Constantin RP, Garcia RF, da Costa $\mathrm{CE}$, et al. Liver mitochondrial function and redox status in an experimental model of non-alcoholic fatty liver disease induced by monosodium 1-glutamate in rats. Exp Mol Pathol 2011;91(3):687-94.

9. Ikeda Y, Fujii J, Taniguchi N, Meister A. Expression of an active glycosylated human gamma-glutamyl transpeptidase mutant that lacks a membrane anchor domain. Proc Natl Acad Sci U S A 1995;92(1):126-30

10. Nakanishi N, Suzuki K, Tatara K. Serum gamma-glutamyltransferase and risk of metabolic syndrome and Type 2 diabetes in middle-aged Japanese men. Diabetes Care 2004;27(6):1427-32.

11. Marchesini G, Brizi M, Bianchi G, Tomassetti S, Bugianesi E, Lenzi M, et al. Nonalcoholic fatty liver disease: A feature of the metabolic syndrome. Diabetes 2001;50(8):1844-50.

12. Gohel MG, Chacko AN. Serum GGT activity and hsCRP level in patients with Type 2 diabetes mellitus with good and poor glycemic control: An evidence linking oxidative stress, inflammation and glycemic control. J Diabetes Metab Disord 2013;12(1):56.

13. Meisinger C, Lowel H, Heier M, Schneider A, Thorand B; KORA Study Group. Serum gamma glutamyl transferase and risk Type 2 diabetes mellitus in men and women from the general population. J Intern Med 2005;258(6):527-35.

14. Lee DH, Silventoinen K, Jacobs DR Jr, Jousilahti P, Tuomileto J. gamma-Glutamyltransferase, obesity, and the risk of Type 2 diabetes: Observational cohort study among 20,158 middle-aged men and women. J Clin Endocrinol Metab 2004;89(11):5410-4.

15. Wannamethee SG, Shaper AG, Lennon L, Whincup PH. Hepatic enzymes, the metabolic syndrome and risk of Type 2 diabetes in older man. Diabetes care 2005;28(12):2913-8.

16. Puukka K, Hietala J, Koivisto H, Anttila P, Bloigu R, Niemelä O. Additive effects of moderate drinking and obesity on serum gammaglutamyl transferase activity. Am J Clin Nutr 2006;83(6):1351-4.

17. Pouliot MC, Després JP, Lemieux S, Moorjani S, Bouchard C, Tremblay A, et al. Waist circumference and abdominal sagittal diameter: Best simple anthropometric indexes of abdominal visceral adipose tissue accumulation and related cardiovascular risk in men and women. Am J Cardiol 1994;73(7):460-8.

18. Kasapoglu B, Turkay C, Bayram Y, Koca C. Role of GGT in diagnosis of metabolic syndrome: A clinical- based cross-sectional survey. Indian J Med Res 2010;132:56-61.

19. Pangaluri R, Akila S, William E. Prevalence of metabolic syndrome and its components in women with subclinical hypothyroidism. AJPCR 2013;6(4):82-4

20. Sabanayagam C, Shankar A, Li J, Pollard C, Ducatman A. Serum gamma-glutamyl transferase level and diabetes mellitus among US adults. Eur J Epidemiol 2009;24(7):369-73.

21. Malnick SD, Beergabel $M$, Knobler $H$. Non-alcoholic fatty liver: A common manifestation of a metabolic disorder. QJM 2003;96(10):699-709.

22. Vinodhini VM, Devisri V, William WE, Akshmi MM, Chandrasekar A, Gnanasambandam S. High sensitive C-reactive protein and apolipoprotein B levels in polycystic ovary syndrome. Int J Pharm Bio Sci 2012;3(2):B719-24

23. Targher G, Seidell JC, Tonoli M, Muggeo M, De Sandre G, Cigolini M. The white blood cell count: Its relationship to plasma insulin and other cardiovascular risk factors in healthy male individuals. J Intern Med 1996;239(5):435-41. 